PROCEEDINGS OF THE AMERICAN MATHEMATICAL SOCIETY

Volume 124, Number 2, February 1996

\title{
INTEGRAL EQUATIONS IN REFLEXIVE BANACH SPACES AND WEAK TOPOLOGIES
}

\author{
DONAL O'REGAN
}

(Communicated by Palle E. T. Jorgensen)

\begin{abstract}
The Schauder Tychonoff theorem in a locally convex topological space is used to establish existence results for Volterra-Hammerstein and Hammerstein integral equations in a reflexive Banach space.
\end{abstract}

\section{INTRODUCTION}

This paper studies integral equations in a reflexive Banach space relative to the weak topology. In particular, in $\S 2$ we establish the existence of a weak solution (described in $\S 2$ ) to the Volterra-Hammerstein integral equation

$$
y(t)=h(t)+\int_{0}^{t} k(t, s) f(s, y(s)) d s, \quad t \in[0, T],
$$

where $T>0$ is fixed. Here $y$ takes values in a reflexive Banach space $B$. The Schauder Tychonoff theorem in locally convex spaces is used to establish existence. Our method has the added advantage in that it discusses automatically the interval of existence $[0, T]$. We note as well that no compactness condition will be assumed on the nonlinearity $f$; this will be due to the fact that a subset of a reflexive Banach space is weakly compact iff it is weakly closed and norm bounded. The results of this section complement related work in the literature; see $[2,5,13,14]$. For example in $[5,13,14]$ some very interesting results for the differential system $y^{\prime}=f(t, y)$ (which is a particular case of the Volterra-Hammerstein equation) are presented. The basic idea in these papers is to use a "successive approximation" type of approach to show "local" existence. The interval of existence from a "construction" point of view is only briefly discussed. Section 3 discusses the Hammerstein integral equation

$$
y(t)=h(t)+\int_{0}^{1} k(t, s) f(s, y(s)) d s, \quad t \in[0,1],
$$

with $y$ taking values in $B$.

For the remainder of this section we gather together some results which will be used throughout this paper. $B$ will always be a reflexive Banach space with norm $\|\cdot\| . B^{*}$ will denote the dual of $B$. We will let $B_{w}$ denote the space $B$ when endowed with the weak topology generated by the continuous linear functionals on $B$ (the family of seminorms $\left\{\rho_{h}: h \in B^{*}\right\}$ is defined by $\rho_{h}(x)=|h(x)|$ for all $x \in B)$.

Received by the editors April 22, 1994 and, in revised form, September 21, 1994. 1991 Mathematics Subject Classification. Primary 45D05, 45G10, 45N05.

(C)1996 American Mathematical Society 
We recall, for convenience $[7,11,12,15]$, the following: Let $y(t)$ be a function from $[a, b]$ into $B$. Then

(i) $y(t)$ is said to be weakly continuous at $t_{0} \in[a, b]$ if for every $\phi \in B^{*}$ we have $\phi(y(t))$ continuous at $t_{0}$.

(ii) $y(t)$ is weakly Riemann integrable on $[a, b]$ if for any partition $\left\{t_{0}, \ldots\right.$, $\left.t_{n}\right\}$ of $[a, b]$ and any choice of points $\tau_{i}, t_{i-1} \leq \tau_{i} \leq t_{i}, i=1, \ldots, n$, the sums $\sum_{i=1}^{n} y\left(\tau_{i}\right)\left(t_{i}-t_{i-1}\right)$ converge weakly to some element $y_{0} \in B$, provided

$$
\max _{1 \leq i \leq n}\left|t_{i}-t_{i-1}\right| \rightarrow 0
$$

i.e. there exists an element $y_{0} \in B$ such that

$$
\phi\left(y_{0}\right)=\int_{a}^{b} \phi(y(s)) d s \quad \text { for all } \phi \in B^{*} .
$$

Similarly we can define the Bochner (respectively Pettis) integral of a strongly (respectively weakly) measurable function $y:[a, b] \rightarrow B$; see $[7,11,12]$. In particular if $y$ is weakly continuous on $[a, b]$, then $y$ is strongly measurable [12]; also if $B$ is reflexive, $y$ is weakly Riemann integrable $[11,13]$.

Now $C\left([a, b], B_{w}\right)$ denotes the family of weakly continuous functions on $[a, b]$ (the family of seminorms $\left\{\eta_{h}\right\}$ is defined by $\eta_{h}(g)=\sup _{x \in[a, b]} \rho_{h}(g(x)$ ) for all $\left.g \in C\left([a, b], B_{w}\right)\right) . C\left([a, b], B_{w}\right)$ is a locally convex topological space; see $[6,8]$.

Next we recall the following results from the literature on functional analysis $[1,2,4,7,13,15]$.

Theorem 1.1 (Schauder Tychonoff). Let $K$ be a closed convex subset of a locally convex (Hausdorff) space E. Assume that $f: K \rightarrow K$ is continuous and that $f(K)$ is relatively compact in $E$. Then $f$ has at least one fixed point in $K$.

Theorem 1.2 (Arzela Ascoli). Let $F$ be a weakly equicontinuous family of functions from $I=[a, b]$ into $B$, and let $\left\{x_{n}(t)\right\}$ be a sequence in $F$ such that for each $t \in I$, the set $\left\{x_{n}(t), n \geq 1\right\}$ is weakly relatively compact. Then there exists a subsequence $\left\{x_{n_{k}}(t)\right\}$ which converges weakly uniformly on $I$ to a weakly continuous function.

Remark. (i) A family $F=\left\{f_{i}, i \in J\right\}, J$ some index set, is said to be weakly equicontinuous if given $\varepsilon>0, \phi \in B^{*}$ there exists $\delta>0$ such that, for $t, s \in[a, b]$, if $|t-s|<\delta$, then

$$
\left|\phi\left(f_{i}(t)-f_{i}(s)\right)\right|<\varepsilon \quad \text { for all } i \in J .
$$

(ii) $\left\{x_{n}(t)\right\}_{n=1}^{\infty}$ converges weakly uniformly on $I$ to a function $x(t)$ if for all $\varepsilon>0$, $\phi \in B^{*}$ there exists an integer $N$ so that $n>N$ implies

$$
\left|\phi\left(x_{n}(t)-x(t)\right)\right|<\varepsilon \quad \text { for all } t \in I .
$$

Theorem 1.3 (Eberlein Šmulian). Suppose $K$ is weakly closed in a Banach space $E$. Then the following are equivalent:

(i) $K$ is weakly compact.

(ii) $K$ is weakly sequentially compact, i.e. any sequence in $K$ has a subsequence which converges weakly.

Theorem 1.4. A subset of a reflexive Banach space is weakly compact iff it is closed in the weak topology and bounded in the norm topology.

Theorem 1.5. A convex subset of a normed space $X$ is closed iff it is weakly closed. 
Finally we state a result which is an immediate consequence of the Hahn Banach theorem.

Theorem 1.6. Let $X$ be a normed space with $0 \neq x_{0} \in X$. Then there exists a $\phi \in X^{*}$ with $\|\phi\|=1$ and $\phi\left(x_{0}\right)=\left\|x_{0}\right\|$.

\section{Volterra integral equations in Reflexive Banach SPACES}

Throughout this section $B$ will be a reflexive Banach space. We will study the Volterra-Hammerstein integral equation

$$
y(t)=h(t)+\int_{0}^{t} k(t, s) f(s, y(s)) d s, \quad t \in[0, T],
$$

where $T>0$ is fixed. Assume

$$
\begin{gathered}
f:[0, T] \times B \rightarrow B \text { is weakly-weakly continuous, } \\
h:[0, T] \rightarrow B \text { is weakly continuous, }
\end{gathered}
$$

and

$$
\left\{\begin{array}{l}
k(t, s) \in L^{1}([0, T], \mathbf{R}) \text { for each } t \in[0, T] \text { and the map } \\
t \rightarrow k(t, s) \text { is continuous from }[0, T] \text { to } L^{1}([0, T], \mathbf{R}) ; \\
\text { also there exists } v \in L^{1}[0, T] \text { and constants } \alpha>0, \\
\beta>0 \text { such that for } x<t \text { in }[0, T] \text { we have } \\
\int_{x}^{t}|k(t, s)| d s \leq \beta\left(\int_{x}^{t} v(s) d s\right)^{\alpha}
\end{array}\right.
$$

are satisfied.

Remark. Let $g:[a, b] \times B \rightarrow B$. Then $g(t, u)$ is said to be weakly-weakly continuous at $\left(t_{0}, u_{0}\right)$ if given $\varepsilon>0, \phi \in B^{*}$ there exists $\delta>0$ and a weakly open set $U$ containing $u_{0}$ such that

$$
\left|\phi\left(g(t, u)-g\left(t_{0}, u_{0}\right)\right)\right|<\varepsilon \quad \text { whenever }\left|t-t_{0}\right|<\delta \text { and } u \in U .
$$

Theorem 2.1. Suppose $f$ satisfies (2.2). Let $\mu>0$ be given and define

$$
Q=\{(t, u): 0 \leq t \leq T,\|u\| \leq \mu\} \subseteq[0, T] \times B .
$$

Then there exists a constant $K_{\mu}>0$ such that $\|f(t, u)\| \leq K_{\mu}$ for all $(t, u) \in Q$.

Proof. Let $V=\{u:\|u\| \leq \mu\} \subseteq B$. Now $V$ is weakly compact from Theorems 1.4 and 1.5. Consequently Tychonoff's theorem implies that $Q$ is compact in the product (i.e. real $\times$ weak) topology. Since $f$ is weakly-weakly continuous on $[0, T] \times B$, we have that the range $f(Q)$ is weakly compact. Consequently Theorem 1.4 implies that $f(Q)$ is bounded in the norm topology.

By a solution to $(2.1)$ we mean a function $y \in C\left([0, T], B_{w}\right)$ which satisfies the integral equation in (2.1). This is equivalent (consequence of the Hahn Banach theorem) to finding a function $y \in C\left([0, T], B_{w}\right)$ with

$$
\phi(y(t))=\phi\left(h(t)+\int_{0}^{t} k(t, s) f(s, y(s)) d s\right), \quad t \in[0, T] \text { for all } \phi \in B^{*} .
$$


Theorem 2.2. Suppose (2.2), (2.3), and (2.4) hold. In addition assume

$$
\left\{\begin{array}{l}
\text { there exist a nondecreasing continuous (independent of } \phi) \text { function } \\
\psi:[0, \infty) \rightarrow(0, \infty) \text { and a constant } \sigma \geq 1 \text { with } \\
\left|\phi\left(\int_{0}^{t} k(t, s) f(s, y(s)) d s\right)\right| \leq \psi\left(\int_{0}^{t}\|y(s)\|^{\sigma} d s\right) \\
\text { for any (norm continuous) } y \in C\left([0, T], B_{w}\right) \text { and any } \\
\phi \in B^{*} \text { with }\|\phi\|=1
\end{array}\right.
$$

and

$$
T<\int_{0}^{\infty} \frac{d u}{\left(\psi(u)+h_{0}\right)^{\sigma}} \quad \text { where } h_{0}=\sup _{[0, T]}\|h(t)\|
$$

are satisfied. Then (2.1) has a solution $y \in C\left([0, T], B_{w}\right)$; in fact the solution we produce will be norm (strongly) continuous.

Proof. Let

$$
J(z)=\int_{0}^{z} \frac{d u}{\left(\psi(u)+h_{0}\right)^{\sigma}}
$$

so $J:[0, \infty) \rightarrow[0, \infty)$ is a strictly increasing function. Also let

$K=\left\{y \in C\left([0, T], B_{w}\right): y\right.$ is norm continuous with $\int_{0}^{t}\|y(s)\|^{\sigma} d s \leq a(t)$

$$
\text { and } \left.\|y\|_{0} \leq M_{0}\right\}
$$

where

$$
a(t)=J^{-1}(t)
$$

and

$$
M_{0}=h_{0}+\psi(a(T)) .
$$

Remark. For notational purposes $\|y\|_{0}=\sup _{[0, T]}\|y(t)\|$.

First notice that $K$ is convex and norm closed. Hence $K$ is weakly closed by Theorem 1.5. Define an operator $N$ by

$$
N y(t)=h(t)+\int_{0}^{t} k(t, s) f(s, y(s)) d s .
$$

We claim that $N: K \rightarrow K$ is weakly continuous and $N(K)$ is weakly relatively compact. Once the claim is established, then Theorem 1.1 with $E=C\left([0, T], B_{w}\right)$ guarantees a fixed point of $N$ in $K$, and hence (2.1) has a solution in $C\left([0, T], B_{w}\right)$.

We begin by showing that $N: K \rightarrow K$. To see this, take $y \in K$ and consider $N y(s)$ for $s \in[0, T]$. Without loss of generality assume $N y(s) \neq 0$ for all $s \in$ $[0, T]$. Then Theorem 1.6 implies that there exists $\phi_{s} \in B^{*}$ with $\left\|\phi_{s}\right\|=1$ and 
$\phi_{s}(N y(s))=\|N y(s)\|$. Thus

$$
\begin{aligned}
\int_{0}^{t}\|N y(s)\|^{\sigma} d s & =\int_{0}^{t}\left(\phi_{s}\left(h(s)+\int_{0}^{s} k(s, x) f(x, y(s)) d x\right)\right)^{\sigma} d s \\
& \leq \int_{0}^{t}\left(\left|\phi_{s}(h(s))\right|+\int_{0}^{s}\left|\phi_{s}(k(s, x) f(x, y(x)))\right| d x\right)^{\sigma} d s \\
& \leq \int_{0}^{t}\left(h_{0}+\psi\left(\int_{0}^{s}\|y(x)\|^{\sigma} d x\right)\right)^{\sigma} d s \\
& \leq \int_{0}^{t}\left(h_{0}+\psi(a(s))\right)^{\sigma} d s=\int_{0}^{t} a^{\prime}(s) d s=a(t)
\end{aligned}
$$

since

$$
\int_{0}^{a(s)} \frac{d x}{\left(\psi(x)+h_{0}\right)^{\sigma}}=s .
$$

Next, we show $\|N y\|_{0}=\sup _{[0, T]}\|N y(t)\| \leq M_{0}$ for any $y \in K$. To see this, look at $N y(t)$ for $t \in[0, T]$. Without loss of generality assume $N y(t) \neq 0$ for all $t \in[0, T]$. Then Theorem 1.6 implies that there exists $\phi_{t} \in B^{*}$ with $\left\|\phi_{t}\right\|=1$ and $\phi_{t}(N y(t))=\|N y(t)\|$. Thus

$$
\begin{aligned}
\|N y(t)\| & =\phi_{t}\left(h(t)+\int_{0}^{t} k(t, s) f(s, y(s)) d s\right) \\
& \leq h_{0}+\int_{0}^{t}\left|\phi_{t}(k(t, s) f(s, y(s)))\right| d s \\
& \leq h_{0}+\psi\left(\int_{0}^{t}\|y(x)\|^{\sigma} d x\right) \leq h_{0}+\psi(a(t)) \leq h_{0}+\psi(a(T))=M_{0} .
\end{aligned}
$$

It remains to show $N y$ is norm continuous for any $y \in K$. To see this, let $t, x \in[0, T]$ with $t>x$, and without loss of generality assume $N y(t)-N y(x) \neq 0$. Then Theorem 1.6 implies that there exists $\phi \in B^{*}$ with $\|\phi\|=1$ and $\phi(N y(t)-N y(x))=$ $\|N y(t)-N y(x)\|$. Notice also since $\|y\|_{0} \leq M_{0}$, then Theorem 2.1 guarantees the existence of a constant $K_{1}$ (independent of the chosen $y$ ) with

$$
\|f(s, y(s))\| \leq K_{1} \quad \text { for all } s \in[0, T] \text { and for all } y \in K .
$$

Thus

$$
\begin{aligned}
\|N y(t)-N y(x)\|= & \phi(N y(t)-N y(x)) \\
\leq & \|h(t)-h(x)\|+K_{1} \int_{0}^{T}|k(t, s)-k(x, s)| d s \\
& +K_{1} \int_{x}^{t}|k(t, s)| d s,
\end{aligned}
$$

so $N y$ is norm continuous. Hence $N: K \rightarrow K$. Also $N: K \rightarrow K$ is weakly continuous. To see this, notice if $y_{n} \rightarrow y$ in $K$ (here $\rightarrow$ denotes weak convergence and $\left(y_{n}\right)$ is a net in $\left.K\right)$, i.e. $y_{n}$ converges weakly uniformly to $y$ on $[0, T]$, then since $f$ satisfies (2.2) we have immediately that $N y_{n}$ converges weakly uniformly to $N y$ on $[0, T]$, so $N$ is weakly continuous.

Next we show that $N(K)$ is weakly relatively compact. To see this, we apply both the Arzela Ascoli and the Eberlein Šmulian theorem. Choose a sequence $y_{n} \in$ 
$K, n \geq 1$. Our aim is to show first that for each $t \in[0, T]$ the set $\left\{N y_{n}(t): n \geq 1\right\}$ is weakly relatively compact. This follows immediately from Theorem 1.4 once we show that for each $t \in[0, T]$ the set $\left\{N y_{n}(t): n \geq 1\right\}$ is norm bounded. For fixed $t \in[0, T]$ we have $\left\|N y_{n}(t)\right\| \leq M_{0}$, so the set $\left\{N y_{n}(t): n \geq 1\right\}$ is weakly relatively compact by Theorem 1.4. Next we show that $N(K)$ is weakly equicontinuous. Let $y \in K$ be arbitrary, and let $t, x \in[0, T]$ with $t>x$. Without loss of generality assume $N y(t)-N y(x) \neq 0$. Then Theorem 1.6 implies that there exists $\phi \in B^{*}$ with $\|\phi\|=1$ and $\phi(N y(t)-N y(x))=\|N y(t)-N y(x)\|$. Also (2.10) is true so

$$
\begin{aligned}
\|N y(t)-N y(x)\|= & \phi\left(h(t)-h(x)+\int_{0}^{x}[k(t, s)-k(x, s)] f(s, y(s)) d s\right. \\
& \left.+\int_{x}^{t} k(t, s) f(s, y) d s\right) \\
\leq & \|h(t)-h(x)\|+K_{1} \int_{0}^{T}|k(t, s)-k(x, s)| d s \\
& +K_{1} \int_{x}^{t}|k(t, s)| d s,
\end{aligned}
$$

and this together with (2.3) and (2.4) implies that $N(K)$ is weakly equicontinuous. Theorem 1.2 guarantees that the weak closure of $N(K)$ is weakly sequentially compact, and this together with Theorem 1.3 implies that the weak closure of $N(K)$ is weakly compact, i.e. $N(K)$ is weakly relatively compact. Theorem 1.1 now guarantees that (2.1) has a solution $y \in K$.

Remarks. (i) Another existence result for (2.1) will be established in $\S 3$.

(ii) Of course (2.5) could be replaced by other growth conditions and existence will again be guaranteed (provided (2.6) is appropriately adjusted).

\section{Hammerstein integral Equations in Reflexive Banach SPACES}

Let $B$ be a reflexive Banach space, and consider the Hammerstein integral equation

$$
y(t)=h(t)+\int_{0}^{1} k(t, s) f(s, y(s)) d s, \quad t \in[0,1],
$$

with

$$
\begin{gathered}
f:[0,1] \times B \rightarrow B \text { is weakly-weakly continuous, } \\
h:[0,1] \rightarrow B \text { is weakly continuous, }
\end{gathered}
$$

and

$$
\left\{\begin{array}{l}
k(t, s) \in L^{1}([0,1], \mathbf{R}) \text { for each } t \in[0,1] \text { and the map } \\
t \rightarrow k(t, s) \text { is continuous from }[0,1] \text { to } L^{1}([0,1], \mathbf{R})
\end{array}\right.
$$

holding.

Theorem 3.1. Suppose (3.2), (3.3), and (3.4) hold. In addition assume

$$
\left\{\begin{array}{l}
\text { there exists a nondecreasing continuous function } \Omega:[0, \infty) \rightarrow(0, \infty) \\
\text { with }\|f(s, u)\| \leq \Omega(\|u\|) \text { for } t \in[0,1]
\end{array}\right.
$$


and

$$
A \equiv\left(\sup _{t \in[0,1]} \int_{0}^{1}|k(t, s)| d s\right) \limsup _{x \rightarrow \infty} \frac{\Omega(x)}{x}<1
$$

are satisfied. Then $(3.1)$ has a solution $y \in C\left([0,1], B_{w}\right)$.

Proof. Consider the set $S$ of real numbers $x \geq 0$ which satisfy the inequality

$$
x \leq \sup _{[0,1]}\|h(t)\|+\Omega(x)\left(\sup _{t \in[0,1]} \int_{0}^{1}|k(t, s)| d s\right) .
$$

Then $S$ is bounded above, i.e. there exists a constant $M_{1}$ with

$$
x \leq M_{1} \text { for all } x \in S .
$$

To see this, suppose (3.7) is not true. Then there exists a sequence $0 \neq x_{n} \in S$ with $x_{n} \rightarrow \infty$ as $n \rightarrow \infty$ and

$$
1 \leq \frac{\sup _{[0,1]}\|h(t)\|}{x_{n}}+\frac{\Omega\left(x_{n}\right)}{x_{n}}\left(\sup _{t \in[0,1]} \int_{0}^{1}|k(t, s)| d s\right) .
$$

Since $\lim \sup \left(s_{n}+t_{n}\right) \leq \lim \sup \left(s_{n}\right)+\lim \sup \left(t_{n}\right)$ for any sequences $s_{n} \geq 0, t_{n} \geq 0$, we have $1 \leq A$. This contradicts (3.6). Choose $M_{0}>M_{1}$. Then

$$
\sup _{[0,1]}\|h(t)\|+\Omega\left(M_{0}\right)\left(\sup _{t \in[0,1]} \int_{0}^{1}|k(t, s)| d s\right)<M_{0},
$$

for otherwise $M_{0} \in S$, and this would contradict (3.7). Let

$$
K=\left\{y \in C\left([0, T], B_{w}\right): y \text { is norm continuous and }\|y\|_{0}=\sup _{[0,1]}\|y(t)\| \leq M_{0}\right\} .
$$

Define an operator $N$ by

$$
N y(t)=h(t)+\int_{0}^{1} k(t, s) f(s, y(s)) d s .
$$

We claim $N: K \rightarrow K$. To see this, let $y \in K$, and consider $N y(t)$ for $t \in[0,1]$. Without loss of generality assume $N y(t) \neq 0$ for $t \in[0,1]$. Then there exists $\phi_{t} \in B^{*}$ with $\left\|\phi_{t}\right\|=1$ and $\phi_{t}(N y(t))=\|N y(t)\|$. Now notice (3.5) and (3.8) imply for each $t \in[0,1]$ that

$$
\begin{aligned}
\|N y(t)\| & \leq\left|\phi_{t}(h(t))\right|+\int_{0}^{1}\left|k(t, s) \phi_{t}(f(s, y(s)))\right| d s \\
& \leq\|h(t)\|+\int_{0}^{1}|k(t, s)| \Omega(\|(y(s))\|) d s \\
& \leq\|h\|_{0}+\Omega\left(\|y\|_{0}\right)\left(\sup _{[0,1]} \int_{0}^{1}|k(t, s)| d s\right) \\
& \leq\|h\|_{0}+\Omega\left(M_{0}\right)\left(\sup _{[0,1]} \int_{0}^{1}|k(t, s)| d s\right)<M_{0} .
\end{aligned}
$$


Thus $N: K \rightarrow K$. Also as in Theorem $2.2 N$ is weakly continuous and $N(K)$ is relatively weakly compact. Theorem 1.1 now guarantees that (3.1) has a solution $y \in K$.

Remark. The ideas in Theorem 3.1 immediately yield an extra existence result for the Volterra integral equation (2.1), namely: suppose (2.2), (2.3), (2.4) with

$$
\left\{\begin{array}{l}
\text { there exists a nondecreasing continuous function } \Omega:[0, \infty) \rightarrow(0, \infty) \\
\text { with }\|f(s, u)\| \leq \Omega(\|u\|) \text { for } t \in[0, T]
\end{array}\right.
$$

and

$$
\left(\sup _{t \in[0, T]} \int_{0}^{t}|k(t, s)| d s\right) \limsup _{x \rightarrow \infty} \frac{\Omega(x)}{x}<1
$$

satisfied. Then (2.1) has a solution $y \in C\left([0, T], B_{w}\right)$.

\section{REFERENCES}

1. C. Corduneanu, Integral equations and stability of feedback systems, Academic Press, New York, 1973. MR 50:10710

2. _ Integral equations and applications, Cambridge Univ. Press, New York, 1991. MR 92h: 45001

3. _ Perturbations of linear abstract Volterra equations, J. Integral Equations Appl. 2 (1990), 393-401. MR 92a:45005

4. J. B. Conway, A course in functional analysis, Springer-Verlag, Berlin, 1990. MR 91e:46001

5. E. J. Cramer, V. Lakshmikantham, and A. R. Mitchell, On the existence of weak solutions of differential equations in nonreflexive Banach spaces, Nonlinear Anal. 2, (1978), 169-177. MR 81d:34051

6. C. De Vito, Functional analysis, Academic Press, New York, 1978. MR 80d:46001

7. N. Dunford and J. T. Schwartz, Linear operators, Interscience, Wiley, New York, 1958. MR 22:8302

8. K. Floret, Weakly compact sets, Lecture Notes in Math., vol. 801, Springer-Verlag, Berlin, 1980. MR 82b:46001

9. G. Gripenberg, S. O. Londen, and O. Staffans, Volterra integral and functional equations, Cambridge Univ. Press, New York, 1990. MR 91c:45003

10. R. B. Guenther and J. W. Lee, Some existence results for nonlinear integral equations via topological transversality, J. Integral Equations Appl. 5 (1993), 195-209. MR 94f:45009

11. E. Hille, Methods in classical and functional analysis, Addison-Wesley, Reading, MA, 1972. MR 57:3802

12. E. Hille and R. S. Phillips, Functional analysis and semigroups, Amer. Math. Soc. Colloq. Publ., vol. 31, Amer. Math. Soc., Providence, RI, 1957. MR 19:664d

13. V. Lakshmikantham and S. Leela, Nonlinear differential equations in abstract spaces, Pergamon Press, Oxford, 1981. MR 82i:34072

14. A. Szep, Existence theorems for weak solutions of ordinary differential equations in reflexive Banach spaces, Studia Sci. Math. Hungar. 6, (1971), 197-203.

15. K. Yosida, Functional analysis, Springer-Verlag, Berlin, 1971.

Department of Mathematics, University College Galway, Galway, Ireland E-mail address: Donal.oRegan@ucg.ie 